\title{
Robust Non-Rigid Registration of 2D and 3D Graphs
}

\author{
Eduard Serradell ${ }^{1}$, Przemyslaw Glowacki ${ }^{2}$, Jan Kybic ${ }^{3}$, Francesc Moreno-Noguer ${ }^{1}$, Pascal Fua ${ }^{2}$ \\ ${ }^{1}$ Institut de Robòtica i Informàtica Industrial (CSIC-UPC), Barcelona, Spain \\ ${ }^{2}$ Computer Vision Laboratory (EPFL), Lausanne, Switzerland \\ ${ }^{3}$ Faculty of Electrical Engineering (CTU), Prague, Czech Republic
}

\begin{abstract}
We present a new approach to matching graphs embedded in $\mathbb{R}^{2}$ or $\mathbb{R}^{3}$. Unlike earlier methods, our approach does not rely on the similarity of local appearance features, does not require an initial alignment, can handle partial matches, and can cope with non-linear deformations and topological differences.

To handle arbitrary non-linear deformations, we represent them as Gaussian Processes. In the absence of appearance information, we iteratively establish correspondences between graph nodes, update the structure accordingly, and use the current mapping estimate to find the most likely correspondences that will be used in the next iteration. This makes the computation tractable.

We demonstrate the effectiveness of our approach first on synthetic cases and then on angiography data, retinal fundus images, and microscopy image stacks acquired at very different resolutions.

\section{Introduction}

Graph-like structures are pervasive in biomedical 2D and 3D images. Examples are blood vessels, pulmonary bronchi, or nerve fibers. They can be acquired at different times and scales, or using different modalities, which may result in vastly diverse image appearances. For example, neuronal structures acquired using a light microscope (LM) such as those on the left of Fig. 1 look radically different when imaged using an electron microscope (EM), as shown on the right of Fig. 1. Nevertheless, registering them is desirable to combine the specific information each modality provides, in this case large-scale connectivity from the low-resolution data and fine details such as dendritic spines from the high-resolution data. Such drastic appearance changes make it impractical to use registration techniques that rely on maximizing image similarity $[26,18]$, in particular when the images are very different and when dealing with thin structures, such as blood ves-
\end{abstract}
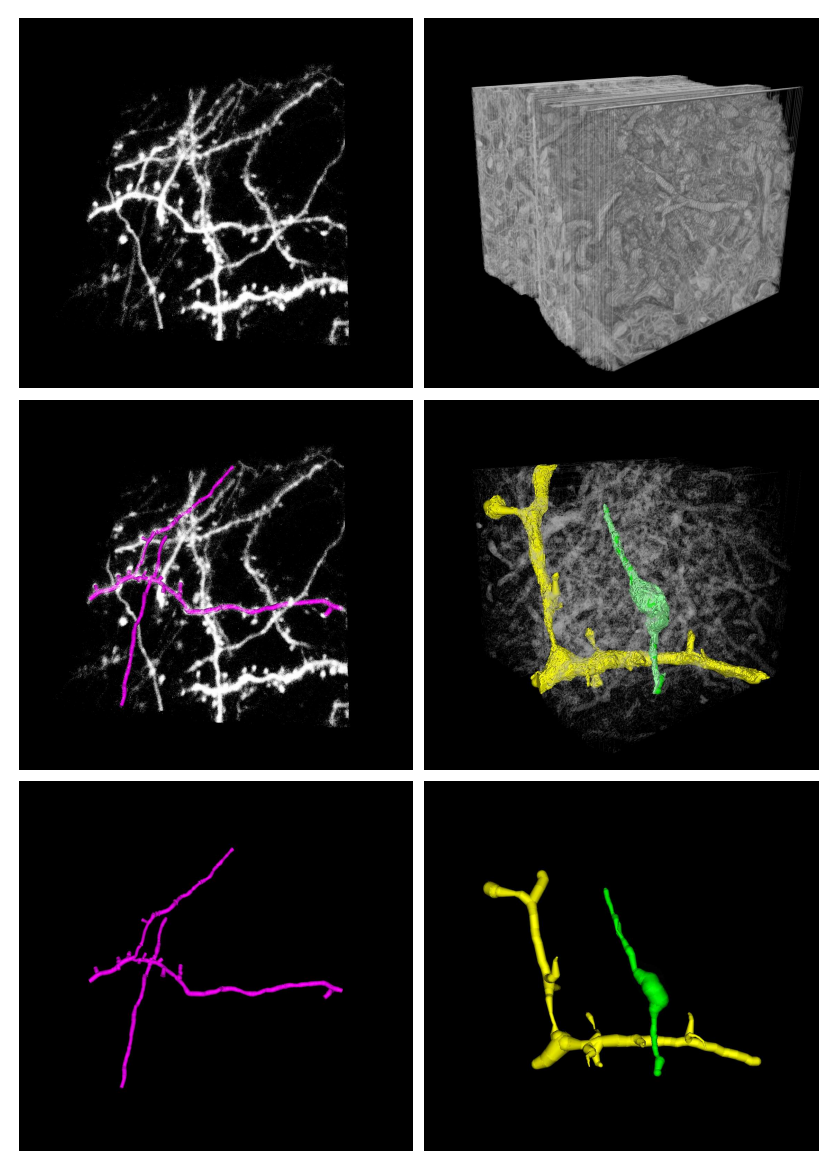

Figure 1. Brain tissue at different resolutions. Top: Left) Image stack acquired using a light microscope from live brain tissue at the micron resolution. Right) A smaller area of the same tissue imaged using an electron microscope after excision and fixation, at a 20 nanometer resolution or 5 times that of the two-photon microscope. Center: Left) Semi-automated delineation of some dendrites overlaid in magenta. Right) Manual segmentation of an axon overlaid in green and a dendrite in yellow. Down: The same structures on a black background. Because the resolution is so much higher in the EM data, dendritic spines and synapses are clearly visible. This figure, as most others in this paper, is best viewed in color. 
sels or neuronal fibers. The lack of distinguishing features of individual branching points or edges makes the use of feature-based correspondence techniques equally impractical. Since the graph geometrical and topological structure may be the only property shared across modalities, graph matching becomes the only effective registration means. This also includes subgraph matching when the images have been acquired at different resolutions.

Most existing techniques that attempt to do this rely on matching Euclidean or Geodesic distance between graph junction points $[9,24,5]$, which is very sensitive to the small length changes inherent to the biological structures we consider. This may be valid for pulmonary vessels, which just undergo smooth deformations, or retinal fundus images that show slight non-linearities -due to the curved surface of the retina- when viewed from different perspectives. Yet, when dealing with images acquired using distinct modalities and at different resolutions, the structures to match exhibit significant topology changes and large nonlinear deformations. Furthermore, we know of no current method that can simultaneously handle non-linear deformation, unknown initial position and lack of distinguishing local features.

We therefore propose a new approach for matching graph structures embedded in either $\mathbb{R}^{2}$ or $\mathbb{R}^{3}$, which can handle these cases while being robust to topological differences between the two graphs and even changes in the distances between vertices. It requires no initial pose estimate, can handle non-linear deformations, and does not rely on local appearance or global distance matrices. Instead, given graphs in the two images or image-stacks to be registered, we treat graph nodes as the features to be matched. We model the geometric mapping from one data set to the other as a Gaussian Process whose predictions are progressively refined as more correspondences are added. These predictions are in turn used to explore the set of all possible correspondences starting with the most likely ones, which allows convergence at an acceptable computational cost even though no appearance information is available.

We demonstrate the effectiveness of our technique at registering angiography and retinal-fundus images acquired at different times and different points of view, as well as neural image-stacks acquired using different modalities.

\section{Related Work}

Area-based registration techniques that maximize image similarity criteria such as correlation or mutualinformation [26, 18] are not applicable in our context as they are not designed to deal with truly different appearances and limited capture ranges. We therefore consider only techniques that match graph structures across images, which can be divided into four main classes. In most cases the branching points (nodes) are extracted and used for matching, while the edges connecting them are often ignored.

In the first class the graphs are assumed to be related by a low-dimensional geometric transform, such as a rigid one, which can be instantiated from very few -only 3 in some cases- correspondences. It is therefore feasible to hypothesize and test random correspondences, as it is done in RANSAC [7] or its variants [4]. However, RANSAC-like approaches quickly become impractical as the number of transformation parameters or graph nodes increases.

The second class of approaches requires a good initial estimate of the transformation to establish an initial estimate of the correspondences, which are then progressively refined. For rigid transformations, one of the earliest such algorithm is the Iterative Closest Point (ICP) method [2], later extended to non-rigid transformations using techniques such as Non-Rigid ICP $[1,13]$, or Coherent Point Drift (CPD) [16]. A good initial estimate is critical as the algorithm will typically fall into a bad local minimum without it.

The third class of methods relies on having a sufficiently discriminative criterion for pairwise compatibility between nodes, such as local appearance descriptors or geometric compatibility between correspondence pairs [9, $11,25,6,12,3]$. Global correspondences between nodes are then estimated using multidimensional optimization schemes such as graduated assignment [9], spectral techniques $[11,25,12]$ or considering the graphs as an absorbing Markov chain [3]. Considering compatibilities as binary tests, the largest consistent set of matches corresponds to the maximum weighted independent set or equivalently the maximum weighted clique [6]. Due to its high computational cost, the method is only applicable to small graphs. Within medical imaging, some authors have exploited the graph matching formulation to register slightly deformed images or volumes such as pulmonary vessels [24], or warping retinal fundus images [5]. While these methods allow a non-parametric formulation of the problem, they cannot be used when appearance information is unavailable and distances vary due to non-linear deformations, which is the case we consider in this paper.

The final class of methods involves simultaneously searching for correspondences and estimating the transformation parameters using a Kalman filter approach [14, 22, $20,23,21]$. As soon as a few initial correspondences have been established, the set of potential correspondences is rapidly reduced, making the search complexity manageable. However, these algorithms, like RANSAC, require an a priori parametric model whose parameters are computed using the correspondences, and thus, cannot generalize to arbitrary deformations. Similar limitations are also shared by methods relying on implicit shape models $[17,8]$. In the Gaussian Process framework we propose, we also progres- 
sively reduce the number of potential correspondences but, in contrast to these previous approaches, no parametric deformation model is required. Instead, the deformation is completely defined by the correspondences and can therefore be completely generic. We will demonstrate that this enlarges significantly the area of applicability and yields better results.

\section{Approach}

In this work, we assume that we are provided with two graphs $\mathcal{G}^{A}=\left(\mathbf{X}^{A}, \mathbf{E}^{A}\right)$ and $\mathcal{G}^{B}=\left(\mathbf{X}^{B}, \mathbf{E}^{B}\right)$ extracted from two images or image-stacks $A$ and $B$, where the Es represent the graphs' edges and the $\mathbf{X}_{\mathrm{s}}$ their nodes that can be either 2D or 3D vertices. Our goal is to use them to find a mapping $m$ from $A$ to $B$ such that $m\left(\mathbf{x}^{A}\right)$ is as close as possible to $\mathbf{x}^{B}$ in the least-squares sense assuming that $\mathbf{x}^{A}$ and $\mathbf{x}^{B}$ are corresponding pixels or voxels. If correspondences between points belonging to the two graphs were given, we could directly use the Gaussian Process (GP) formalism to compute the mapping as a non-linear regressor [19] that yields a mean prediction $m$ and its associated variance. In our case, however, the correspondences are initially unavailable and cannot be established on the basis of local image information because the $A$ and $B$ are too different in appearance. In short, this means that we must only rely on geometrical properties to simultaneously establish the correspondences and estimate the underlying non-linear transform. To this end, our algorithm goes through the following steps:

1. Coarse alignment: We begin by matching graph nodes so that distances along the edges connecting corresponding nodes, which we will refer to as geodesic distances, are changed as little as possible.

We initialize the process by randomly picking two correspondences, which roughly fixes relative scale and orientation, and use them to instantiate a GP. We then refine it using the recursive procedure described below. If this procedure fails, we pick another random pair until it succeeds.

Given some correspondences between $\mathcal{G}^{A}$ and $\mathcal{G}^{B}$ nodes, the GP serves to predict where other $\mathcal{G}^{A}$ nodes should map and restricts the set of potential correspondences, especially given the fact that geodesic distances must be preserved. Among these possibilities, we select the most likely one, use it to refine the GP, and iterate. Repeating this procedure recursively until enough mutually consistent correspondences have been established and backtracking when necessary lets us quickly explore the set of potential correspondences and recover a rough mapping.

2. Fine alignment: The mapping discussed above has been learned only from the graph nodes (branching points), and is therefore coarse. To refine it, we also establish correspondences between points lying on edges connecting graph nodes. Because there are many more of those than they were nodes, this would be combinatorially explosive if we did it from scratch as done previously. Instead, we constrain the correspondences to be between samples belonging to edges of already connected corresponding nodes and rely on a Hungarian algorithm [15] to perform the optimal assignment quickly.

In the remainder of this section, we first outline the GP formalism that we use. We then discuss our procedures for coarse and fine alignments.

\subsection{Non-Linear Regression}

Consider the case where we are given $N$ correspondences $\left(\mathbf{x}_{i}^{A}, \mathbf{x}_{i}^{B}\right)_{1 \leq i \leq N}$ between $2 \mathrm{D}$ or $3 \mathrm{D}$ points from $A$ and $B$ respectively. Using the GP approach to non-linear regression and assuming Gaussian i.i.d. noise of precision $\beta^{-1}$ in the coordinate values, these correspondences can be used to predict that the point $\mathbf{x}^{B}$ in $B$ corresponding to $\mathbf{x}^{A}$ in $A$ can be expected to be found at a location whose mean $m_{N}$ and variance $\sigma_{N}$ can be computed as

$$
\begin{aligned}
m_{N}\left(\mathbf{x}^{A}\right) & =\mathbf{k}^{T} \mathbf{C}_{N}^{-1} \mathbf{X}_{N}^{B} \\
\sigma_{N}^{2}\left(\mathbf{x}^{A}\right) & =k\left(\mathbf{x}^{A}, \mathbf{x}^{A}\right)+\beta^{-1}-\mathbf{k}^{T} \mathbf{C}_{N}^{-1} \mathbf{k},
\end{aligned}
$$

where $k$ is a kernel function, $\beta$ is proportional to the expected noise-level in the data, $\mathbf{C}_{N}$ is the $N \times N$ symmetric matrix with elements $C_{i, j}=k\left(\mathbf{x}_{i}^{A}, \mathbf{x}_{j}^{A}\right)+\beta^{-1} \delta_{i, j}, \mathbf{k}$ is the vector $\left[k\left(\mathbf{x}_{1}^{A}, \mathbf{x}^{A}\right), \ldots, k\left(\mathbf{x}_{N}^{A}, \mathbf{x}^{A}\right)\right]^{T}$, and $\mathbf{X}_{N}^{B}$ is the vector $\left[\mathbf{x}_{1}^{B}, \ldots, \mathbf{x}_{N}^{B}\right]^{T}$.

Among the different types of kernel functions [19] we chose the widely used summation of a squared-exponential, a constant term, and a linear one

$$
k\left(\mathbf{x}_{i}, \mathbf{x}_{j}\right)=\boldsymbol{\theta}_{0}+\boldsymbol{\theta}_{1} \mathbf{x}_{i}^{T} \mathbf{x}_{j}+\boldsymbol{\theta}_{2} \exp \left\{-\frac{\boldsymbol{\theta}_{3}}{2}\left\|\mathbf{x}_{i}-\mathbf{x}_{j}\right\|^{2}\right\}
$$

We found this kernel to be the most appropriate for our purposes, because it implicitly defines a mapping function composed of an affine plus a non-linear transformation. This accounts for most of the warps appearing in biomedical imaging.

Given this expression for $k$, the mean prediction of Eq. 1 can now be rewritten as

$$
\begin{aligned}
m_{N}\left(\mathbf{x}^{A}\right)= & \sum_{i=1}^{N} a_{i} k\left(\mathbf{x}_{i}^{A}, \mathbf{x}^{A}\right) \\
= & \sum_{i=1}^{N} a_{i}\left(\boldsymbol{\theta}_{0}+\boldsymbol{\theta}_{1}\left(\mathbf{x}_{i}^{A}\right)^{T} \mathbf{x}^{A}\right)+ \\
& \sum_{i=1}^{N} a_{i} \boldsymbol{\theta}_{2} \exp \left\{-\frac{\boldsymbol{\theta}_{3}}{2}\left\|\mathbf{x}_{i}^{A}-\mathbf{x}^{A}\right\|^{2}\right\}
\end{aligned}
$$


Initial graphs

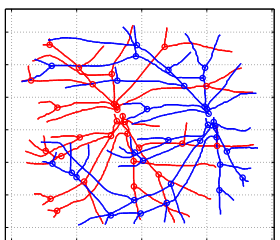

Initialization

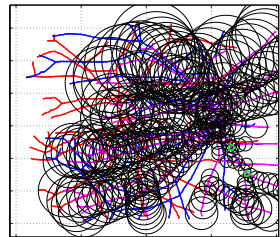

it\#2

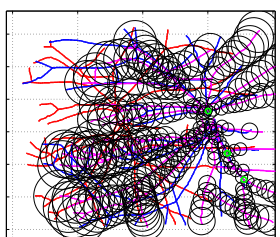

it\#14

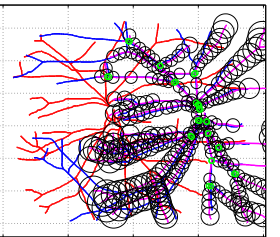

it\#21

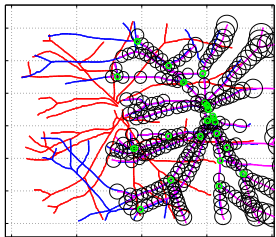

Output 1st step

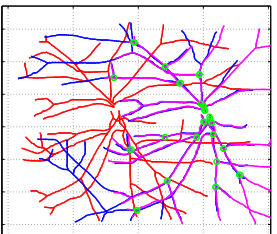

Figure 2. Coarse alignment steps. The initial graph structures are depicted in the left-most figure, the model graph in red and the target in blue. Exploration of the search space starts by picking randomly two correspondences, highlighted in green, thus roughly fixing scale and orientation. Then, the next match candidate is chosen among the nodes located inside the bounded regions, which are a function of the GP predicted covariances, shown as black ellipses. Every correspondence added to the hypotheses set helps refining the mapping uncertainty. The final correspondence set, defines a coarse alignment of the graphs, overlaid in magenta. Best viewed in color.

where $a_{i}$ is the $i^{t h}$ element of the vector $\mathbf{C}_{N}^{-1} \mathbf{X}_{N}^{B}$. The first term of Eq. 4, containing the $\boldsymbol{\theta}_{0}$ and $\boldsymbol{\theta}_{1}$ hyperparameters, is a linear function of the input variables while the second one, involving the $\boldsymbol{\theta}_{2}$ and $\boldsymbol{\theta}_{3}$ hyperparameters and an exponential, allows for additional non-linear deformations.

\subsection{Coarse Alignment}

Let $\mathbf{X}^{A}=\left\{\mathbf{x}_{1}^{A}, \ldots, \mathbf{x}_{n_{A}}^{A}\right\}$ and $\mathbf{X}^{B}=\left\{\mathbf{x}_{1}^{B}, \ldots, \mathbf{x}_{n_{B}}^{B}\right\}$ be the nodes of our two graphs. As discussed at the beginning of this section, our first goal is to simultaneously retrieve as many correspondences $\left\{\mathbf{x}^{A} \leftrightarrow \mathrm{x}^{B}\right\}$ as possible and the underlying non-linear mapping $\mathbf{x}^{B}=m\left(\mathbf{x}^{A}\right)$ that best aligns them.

We take $m$ to be a GP written using the formalism of Section 3.1, which we instantiate by first randomly selecting only two matches. This gives us an initial correspondence set $H_{0}$, enough to roughly fix the global scale and rotation, and then we recursively add new correspondences as follows.

1. Given $k$ correspondences, we compute the mapping $m_{k}($.$) and covariance estimator \sigma_{k}^{2}($.$) of Eqs. 1$ and 2.

2. For each unmatched node $\mathbf{x}_{i}^{A} \in \mathbf{X}^{A}$, we search for potential correspondences $\mathbf{x}_{j}^{B} \in \mathbf{X}^{B}$ in the bounded region defined by the predicted covariance $\sigma_{k}^{2}\left(\mathbf{x}_{i}^{A}\right)$.

3. We choose the node $\mathbf{x}_{i}^{A}$ with the smallest number of potential correspondences, and randomly pick one of them to define the match $\mathbf{x}_{i}^{A} \leftrightarrow \mathbf{x}_{j}^{B}$, which we add to the correspondence set $H_{0}$.

This iterative process is repeated until a large enough $H_{0}$ is found, as is done in RANSAC. If that does not happen, the algorithm backtracks and selects different correspondences using a depth-first search strategy. This is depicted in Fig. 2.

The process is controlled by the vector $\Theta=$ $\left\{\boldsymbol{\theta}_{0}, \boldsymbol{\theta}_{1}, \boldsymbol{\theta}_{2}, \boldsymbol{\theta}_{3}\right\}$ containing the kernel hyperparameters of Eq. 3 and the noise parameter $\beta$ of Eq. 2. To avoid having to tune these parameters for each new dataset, we center and scale the $\mathbf{X}^{A}$ and $\mathbf{X}^{B}$ coordinates so that their average distances to the origin is one and perform the computation on the scaled versions. As a result, we were able to use the same $\Theta$ and $\beta$ for all experiments described in Section 4.

To speed up the computation, we reject correspondences that would produce overly large changes in geodesic distances, which we define as the length $\gamma_{i j}$ of a path connecting the edges between two graph nodes $\mathbf{x}_{i}$ and $\mathbf{x}_{j}$. Given $N$ already established correspondences between graphs, for each new potential match, the geodesic distances connecting the new corresponding points to the nodes in both graphs have to be proportional. We set the tolerance to geodesic distance variations depending on the level of deformations we expect to recover. Proceeding in this way, the algorithm gains robustness against outliers, while it avoids unnecessary checks, thus keeping a low complexity. Note that geodesic distances are invariant to rotations, to the bending of the branches, and to isometric changes.

\subsection{Fine Alignment}

Having performed the rough alignment as described above, we now have an initial set of $N$ correspondences $H_{0}$ between graph nodes, as well as the corresponding GP mapping $m_{N}($.$) and covariance estimator \sigma_{N}^{2}($.$) . Since the$ graph nodes are connected by paths, we can refine the mapping by also establishing correspondences between points that lie on these paths. Since allowing any point to potentially be matched to any other would be prohibitively expensive, we assume the correspondences between nodes to be correct and only establish new ones between points lying on paths linking matching vertices. We again do this iteratively using the following two steps:

1. For each pair of paths connecting pairs of corresponding vertices, we use the Hungarian algorithm [15] to establish matches between the points forming the path. To this end, we use the Mahalanobis distance between potential assignments computed using the current mapping $m($.$) and covariance estimator \sigma^{2}($.$) . All$ the matches need to have consistent geodesic distances to the nodes in their respective graphs.

2. Given these new correspondences, we reestimate $m($. and $\sigma^{2}($.$) and iterate.$ 
it\#1

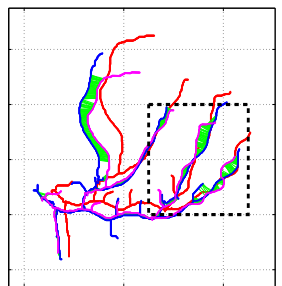

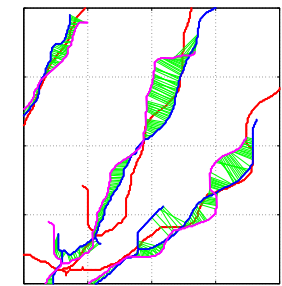

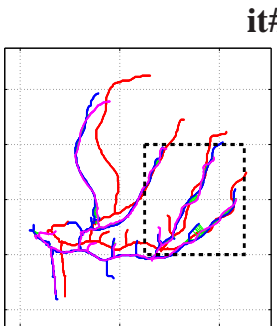

it\#3

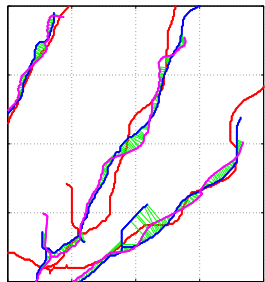

it\#5

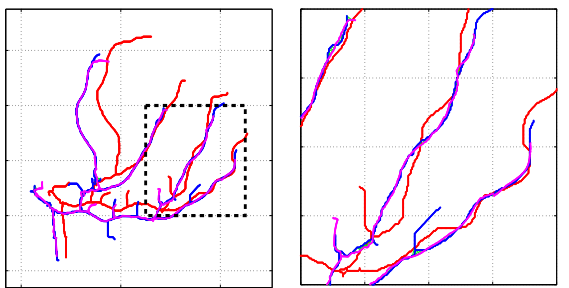

Figure 3. Fine alignment steps. Once a coarse alignment of the two graphs (model in red and target in blue) has been found, the algorithm starts to match points lying on the edges. The assignments (depicted in green) are computed using the Hungarian algorithm and constrained by the graph topology and GP predictions. After a few iterations, the warped structure (magenta) is completely aligned to the target graph. For each successive plot, we zoom to a smaller region to better show the algorithm at work. Best viewed in color.

Eventually, the distance error between both graphs can not be further minimized and the process ends. This yields a final expanded set of correspondences $H_{R}$ and corresponding $m_{R}($.$) and covariance estimator \sigma_{R}^{2}($.$) . Note that we$ use the same GP parameters $\Theta$ and $\beta$ as before. The whole process is illustrated by Fig. 3.

\section{Experiments}

We now present the results on both synthetic and real data, by first extensively evaluating our algorithm in controlled experiments with known ground truth and then showing the results in registering real $2 \mathrm{D}$ and $3 \mathrm{D}$ biomedical images. For the synthetic experiments we compare our approach (denoted Non-Linear GP) to the Coherent Point Drift $(C P D)$ [16], which is a representative example of the state-of-the-art in non-rigid point matching and shape recovery. We also compare it against [21], which uses a Kalman filter based approach to learn an initial affine transform and refines the output with a local non-linear warping. We refer this approach as Affine Kalman.

\subsection{Synthetic Data}

We applied all algorithms to synthetic 3D structures with increasing levels of deformation and amount of outliers. We used Vascusynth[10] to synthesize several 3D trees of 25 nodes each within a volume of $100 \times 100 \times 100$ voxels, such as the one shown in Fig. 4. We simulated the difficulties encountered with real data, by adding increasing levels of noise $\sigma_{n}$ to the tree node locations, bending the branches and introducing spurious nodes and corresponding branches, which we will refer to as outliers. The magnitude of the bending was controlled through a parameter $p_{b}$, which establishes the length ratio between the original edge and the deformed one. The percentage of outliers $p_{o}$ refers to the number of introduced non-corresponding nodes with respect to the original number in the rigid tree. We then registered the original reference shape and the synthetic deformed graphs using each of the algorithms.

Two different types of experiments were performed. We first evaluated the amount of deformation each algorithm was able to recover by sweeping the variances of the accumulative joint noise within the range $\sigma_{n} \in[0-1]$ voxels, and fixing $p_{b}=25 \%$ and $p_{o}=30 \%$. To give significance to these levels of deformation and reconstruction errors, Fig. 4-right depicts different deformations of the reference model corresponding to specific values of $\sigma_{n}$ and $p_{b}$. In a second experiment we computed the robustness to outliers by synthetically introducing random nodes -which turned to outlier tree branches- within the range $p_{o} \in[0-100] \%$, and setting $\sigma_{n}=0.3$ voxels and $p_{b}=25 \%$.

For each set of experimental parameters, we performed 10 trials and compared our approach to the Affine Kalman, and to the CPD both in its affine and non-linear versions. For a fair comparison, since the CPD allows for control of the amount of non-linearity by tuning some internal parameters, we tried several configurations and retained the solution yielding the best results. The graphs on Fig. 4 depict the mean 3D reconstruction error, expressed in voxels, both for increasing deformation levels, and increasing number of outliers. Observe that our algorithm consistently outperforms CPD in all experiments. This demonstrates the advantage of using the geodesic distance compatibility between points, which is inherently used in our approach.

We also clearly outperform [21], mainly for large levels of deformation. While our non-linear algorithm is able to warp the graph while searching for matching nodes, the affine search of [21] only yields reasonable results for low levels of deformation. In addition, observe that the magnitudes of error obtained by our approach are in fact very good approximations.

Finally, we compared against [12], which is representative of the graph matching algorithms. Note that these kind of approaches only tackle the problem of assigning the correspondences, but are not specifically designed for recovering the underlying transformation. We therefore focused only on the retrieved matches. We observed that [12] is only effective when all inlier correspondences show a similar pattern, but has more difficulties under non-linear deformations, as shown in Fig. 5. 
Deformation Level $\left[\sigma_{n}\right]$

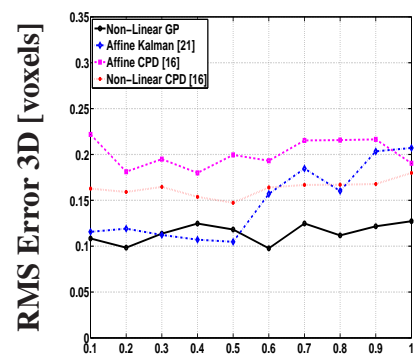

Outliers (\%)

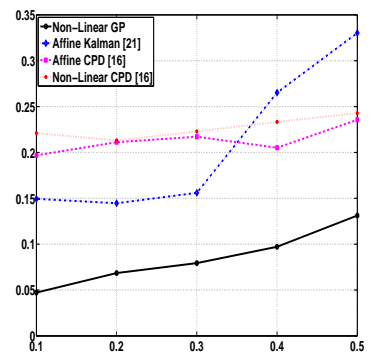

Deformation Samples

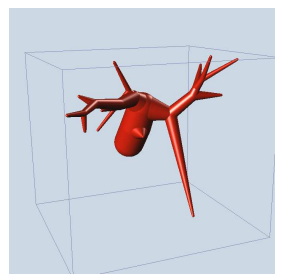

Synthetic

Rigid Tree

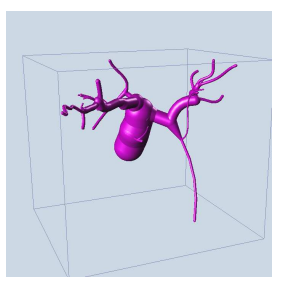

Bended Sample

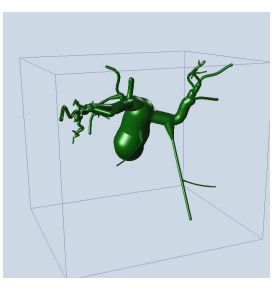

With outliers

Figure 4. Synthetic experiments. Left: RMS error for 3D reconstruction for each experiment. The plot compares the median of the result for each algorithm. Right: Synthetic samples generated using different configurations of the control parameters.
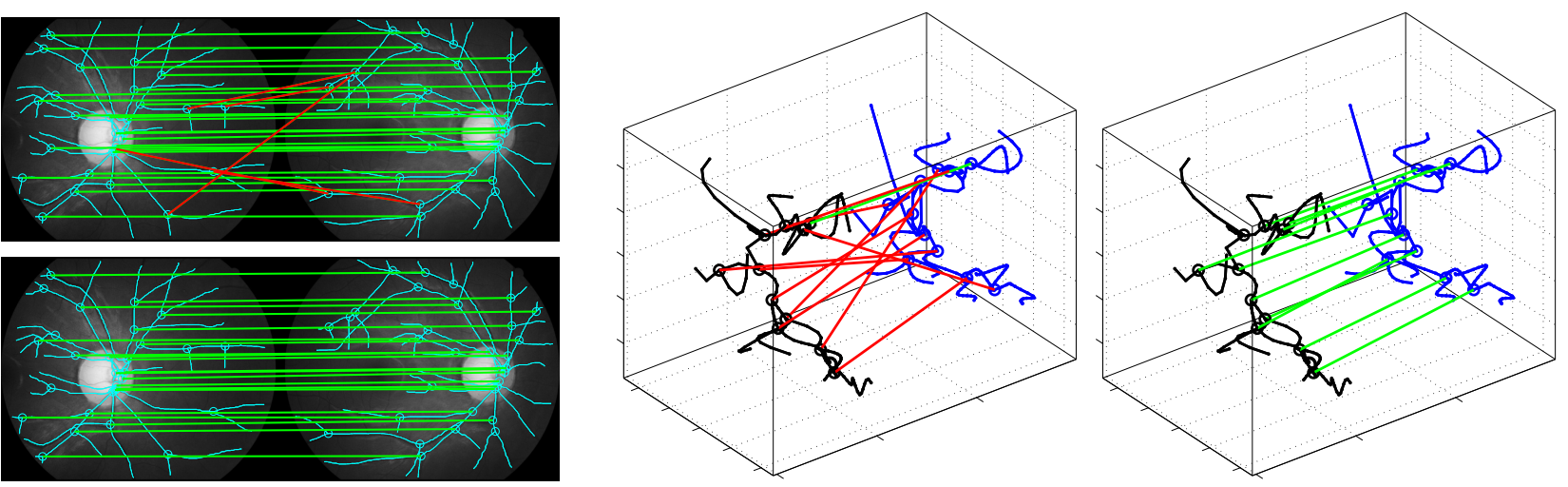

Figure 5. Search for correspondences. Graph matching methods are a common technique to find correspondences. Left: In the retinal fundus images were the deformation is quasi-affine, both the graph matching algorithm [12] (top) and our method (down) were able to recover the correct matching. Right: However, when the structures to register present a too large deformation, the graph matching method (left) missed most of the correct assignments, while our method is still successful (right). Best viewed in color: the correct correspondences are painted in green and the outlier ones in red.

\subsection{Real Data}

We next present some real examples of the results obtained by our algorithm on several biomedical datasets. The graphs were extracted semi-automatically using the Fiji 1 platform and its plugins.

In Fig. 6 we show registration results for retinal fundus vascular graphs that are deformed from one image to the next because the camera is looking from different points of view. This results in apparent distortions of the curved retinal surface's projection, which are well modeled by an affine transform. Because there is very little non-linearity in the deformation, these results are similar to those of [5], even though the trees only partially overlap. However, as the amount of spurious branches is quite large, CPD fails to recover the correct shape. In contrast, our Non-Linear GP can naturally handle such artifacts.

In the 2D X-ray angiography images of Fig. 7 the deformations are much more non-linear. As shown on the zoomed area, our algorithm nevertheless does a good job of recovering this more complex deformation and aligning the trees. Again, we assessed the performance of the CPD on

\footnotetext{
${ }^{1}$ http://pacific.mpi-cbg.de
}

\begin{tabular}{|l|l|l|l|}
\hline Image (Fig.\#) & Image Size & Error NLGP & Error CPD \\
\hline Angio. ( 7 a) & $512 \times 512$ pix & 1.1969 pix & 3.1633 pix \\
\hline Angio. ( 7 b) & $512 \times 512$ pix & 1.8080 pix & 3.4183 pix \\
\hline Ret. ( 6 a) & $1548 \times 1260$ pix & 2.6757 pix & 20.6653 pix \\
\hline Ret. ( 6 b) & $1548 \times 1260$ pix & 2.5109 pix & 20.4496 pix \\
\hline Neuronal ( 8) & $4.4 \times 5.7 \times 6.0 \mu m$ & $0.0702 \mu m$ & $0.2628 \mu m$ \\
\hline
\end{tabular}

Table 1. Comparison of our Non-Linear GP (NLGP) and the Coherent Point Drift (CPD) for real data.

these images and observed that it could not retrieve a correct solution unless a relatively accurate initialization was provided. And even when we supplied our affine estimate, CPD only succeeded when dealing with small non-linearities, but not otherwise, as shown in Fig. 7.

Finally, we register the 3D neuronal stacks extracted from the brain tissue of Fig. 1 using two different modalities. Even though the two images look extremely different, our algorithm returns a valid deformation as shown in Fig. 8. Neither CPD nor Affine Kalman were able to recover the correct alignment.

In order to quantitatively compare the CPD accuracy against ours in the absence of ground truth, we computed an optimal assignment for each node in the deformed graphs 

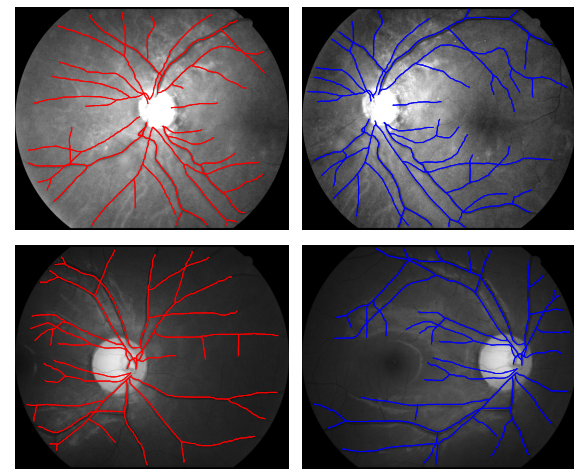

(a)
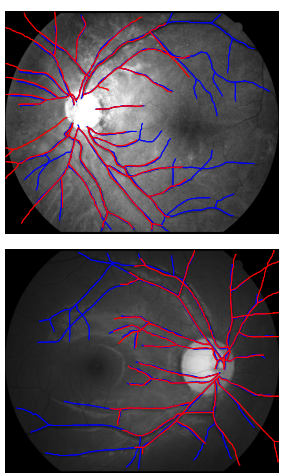

(c)
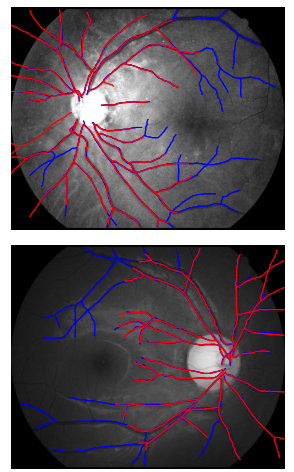

(d)
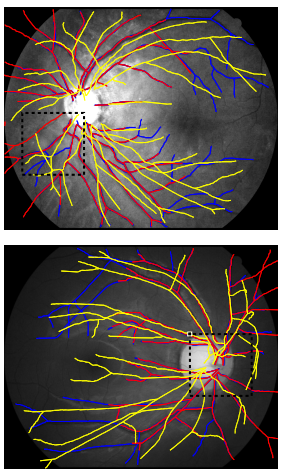

(e)
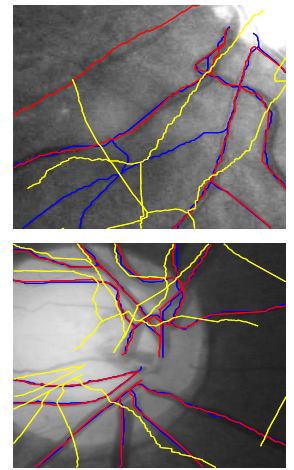

(f)

Figure 6. Retinal fundus images used in [5]. (a,b) Two images of the same retina taken from different viewpoints, with the vascular trees overlaid in red and blue. (c) The first tree is overlaid in red over the second image after non-linear transformation, which corresponds to the output of the coarse alignment. (d) Final result of our non-rigid registration: the graph from the first image is overlaid in red over the second image. (e,f) Our result is superposed with the Coherent Point Drift alignment. In this dataset, our algorithm behave well, but CPD fails to recover the correct shape because of there are too many non-correponding branches. Best viewed in color.
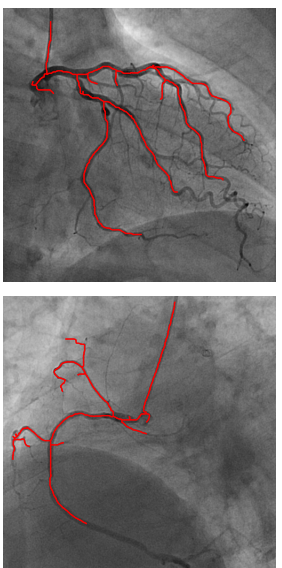

(a)
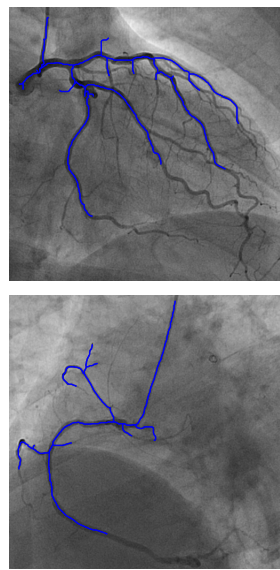

(b)
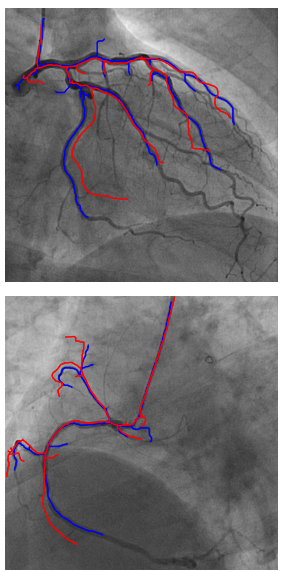

(c)
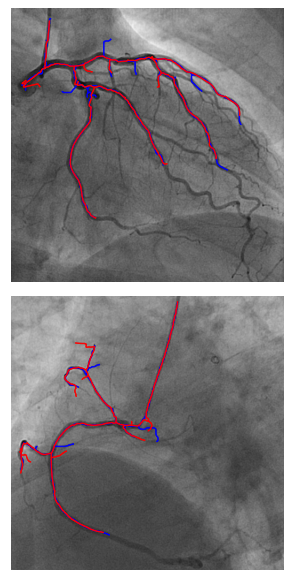

(d)
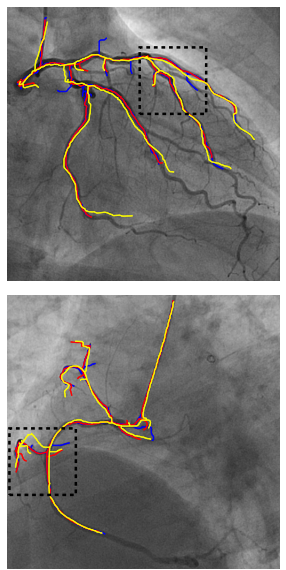

(e)
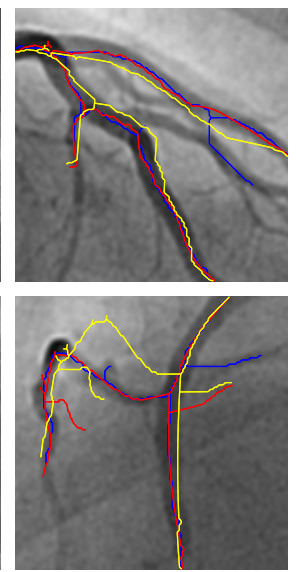

(f)

Figure 7. Angiography images from a beating heart. (a) Two different images with extracted vascular trees overlaid in red. (b) Two other images taken later in the heart cycle with extracted vascular trees overlaid in blue. (c) The original red trees are shown after the non-linear coarse alignment of the tree nodes. (d) The resulting warped trees are overlaid in red after non-linear registration. Note that the trees -in particular in the first example- have distinctly different topologies, which affects our algorithm very little. (e) Comparison with the result obtained using non-linear Coherent Point Drift, in yellow. (f) A zoom of a region of interest. Using the graph intrinsic geometry grants us robustnees against vessel bendings and outliers, achieving a better registration of the two shapes. Best viewed in color.

- overlaid in red for our method and yellow for CPD on Figs. 6 and 7 (d,e,f) - to its nearest neighbor in the corresponding graph overlaid in blue. We report the results of this approximate error in table 1.

\section{Conclusion}

We have shown that our algorithm can match graphs with neither appearance information nor initial pose estimate, while allowing for partial matches and non-linear deformations. This is made possible by using Gaussian Processes to model the mapping from one graph to another and using this mapping to progressively constrain the search area for correspondences between graph nodes.

We have demonstrated our algorithm on graphs contain- ing up to 100 nodes, for which the computation takes approximately 500 seconds in MATLAB on a 8 Core $1.6 \mathrm{GHz}$ 64-bit Processor. A C implementation would produce a significant speedup. A further one will result from refining the strategy we currently use to explore the set of possible correspondences, which is what we are currently working on.

\section{Acknowledgments}

This work has been partially funded by the ERC projects MicroNano and GARNICS FP7-247947, the Spanish Ministry of Economy and Competitiveness under projects PAU+ DPI2011-27510 and MIPRCV Consolider Ingenio 2010 CSD2007-00018, and the Czech Science Foundation under project P202/11/0111. We thank the project 'La Marató' de TV3 082131 for providing the heart dataset. 


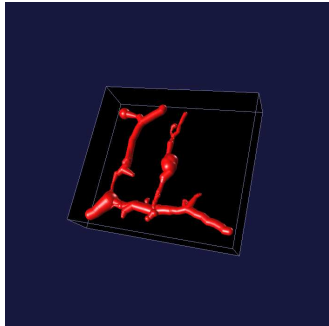

(a)

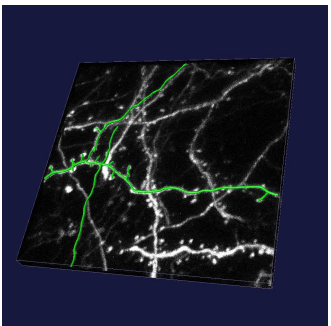

(b)

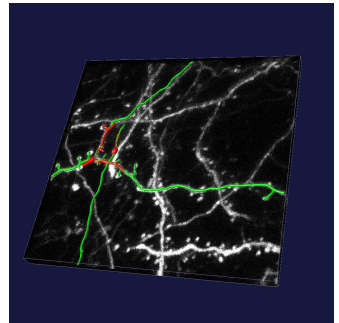

(c)

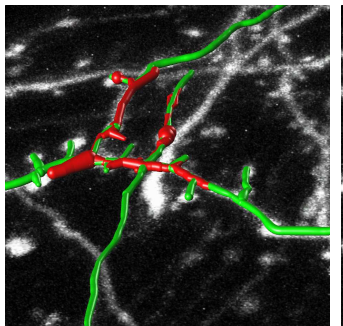

(d)

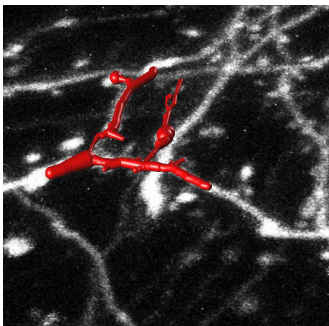

(e)

Figure 8. Neuronal image stacks: multimodal registration. (a) Graph structure extracted from the EM image stack, in red. (b) The segmented graph structure in green, overlaid over the LM image stack. (c) After the non-linear registration process, the EM segmented neuron is deformed and aligned over the LM extracted neuron. (d) A zoom over the region where the EM stack has been extracted. The two neurons have been completely aligned. (e) The EM neuron, embedded in the LM image stack. Best viewed in color.

\section{References}

[1] B. Amberg, S. Romdhani, and T. Vetter. Optimal Step Nonrigid ICP Algorithms for Surface Registration. In CVPR, 2007.

[2] P. Besl and N. Mckay. A Method for Registration of 3D Shapes. IEEE Transactions on Pattern Analysis and Machine Intelligence, 14(2):239-256, February 1992.

[3] M. Cho, J. Lee, and K. Lee. Reweighted Random Walks for Graph Matching. In ECCV, pages 492-505, 2010.

[4] O. Chum and J. Matas. Matching with PROSAC - Progressive Sample Consensus. In $C V P R$, pages 220-226, June 2005.

[5] K. Deng, J. Tian, J. Zheng, X. Zhang, X. Dai, and M. Xu. Retinal Fundus Image Registration via Vascular Structure Graph Matching. International Journal of Biomedical Imaging, 2010.

[6] O. Enqvist, K. Josephson, and F. Kahl. Optimal Correspondences from Pairwise Constraints. In ICCV, 2009.

[7] M. Fischler and R. Bolles. Random Sample Consensus: A Paradigm for Model Fitting with Applications to Image Analysis and Automated Cartography. Communications ACM, 24(6):381-395, 1981.

[8] K. Fujiwara, K. Nishino, J. Takamatsu, B. Zheng, and K. Ikeuchi. Locally Rigid Globally Non-Rigid Surface Registration. In ICCV, 2011.

[9] S. Gold and A. Rangarajan. A Graduated Assignment Algorithm for Graph Matching. IEEE Transactions on Pattern Analysis and Machine Intelligence, 18:377-388, 1996.

[10] P. Jassi and G. Hamarneh. VascuSynth: Vascular Tree Synthesis Software, 2011. http://hdl.handle.net/10380/3260.

[11] M. Leordeanu and M. Hebert. A Spectral Technique for Correspondence Problems Using Pairwise Constraints. In ICCV, pages 1482-1489, October 2005.

[12] M. Leordeanu, M. Hebert, and R. Sukthankar. An Integer Projected Fixed Point Method for Graph Matching and MAP Inference. In Advances in Neural Information Processing Systems, pages 1114-1122, 2009.

[13] H. Li, R. Sumner, and M. Pauly. Global Correspondence Optimization for Non-Rigid Registration of Depth Scans. In Symposium on Geometry Processing, pages 1421-1430, 2008.
[14] F. Moreno-Noguer, V. Lepetit, and P. Fua. Pose Priors for Simultaneously Solving Alignment and Correspondence. In ECCV, October 2008.

[15] J. Munkres. Algorithms for the Assignment and Transportation Problems. Journal of the Society of Industrial and Applied Mathematics, 5(1):32-38, March 1957.

[16] A. Myronenko and X. Song. Point-Set Registration: Coherent Point Drift. IEEE Transactions on Pattern Analysis and Machine Intelligence, 32(12):2262-2275, 2010.

[17] N. Paragios, M. Rousson, and V. Ramesh. Non-Rigid Registration Using Distance Functions. Computer Vision and Image Understanding, pages 142-165, 2003.

[18] J. P. W. Pluim, J. B. A. Maintz, and M. A. Viergever. Mutual Information Based Registration of Medical Images: A Survey. IEEE Transactions on Medical Imaging, 22(8):9861004, 2003.

[19] C. E. Rasmussen and C. K. Williams. Gaussian Process for Machine Learning. MIT Press, 2006.

[20] J. Sanchez-Riera, J. Ostlund, P. Fua, and F. Moreno-Noguer. Simultaneous Pose, Correspondence and Non-Rigid Shape. In $C V P R$, June 2010.

[21] E. Serradell, F. Moreno, J. Kybic, and P. Fua. Robust Elastic 2D/3D Geometric Graph Matching. In SPIE Medical Imaging, 2012.

[22] E. Serradell, M. Özuysal, V. Lepetit, P. Fua, and F. MorenoNoguer. Combining Geometric and Appearance Priors for Robust Homography Estimation. In ECCV, pages 58-72, September 2010.

[23] E. Serradell, A. Romero, R. Leta, C. Gatta, and F. MorenoNoguer. Simultaneous Correspondence and Non-Rigid 3D Reconstruction of the Coronary Tree from Single X-Ray Images. In ICCV, November 2011.

[24] D. Smeets, P. Bruyninckx, J. Keustermans, D. Vandermeulen, and P. Suetens. Robust Matching of 3D Lung Vessel Trees. In MICCAI Workshop on Pulmonary Image Analysis, 2010.

[25] L. Torresani, V. Kolmogorov, and C. Rother. Feature Correspondence via Graph Matching: Models and Global Optimization. In ECCV, pages 596-609, 2008.

[26] B. Zitova and J. Flusser. Image Registration Methods: A Survey. Image and Vision Computing, 11(11):977-1000, 2003. 